\title{
STRAIN-MEDIATED ELECTRICAL CONTROL OF MAGNETIZATION IN MICRON-SCALE NICKEL RING ON PMN-PT
}

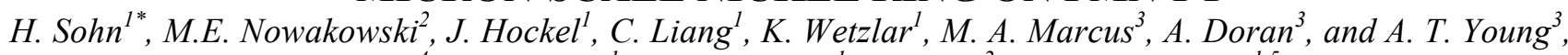

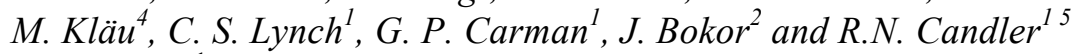 \\ ${ }^{1}$ University of California, Los Angeles, USA \\ ${ }^{2}$ University of California, Berkeley, USA \\ ${ }^{3}$ Lawrence Berkeley National Lab, Berkeley, USA \\ ${ }^{4}$ Johannes Gutenberg-Universität, Mainz, Germany \\ ${ }^{5}$ California NanoSystems Institute, Los Angeles, USA
}

\begin{abstract}
The magnetic "onion" state in a micron-scale ferromagnetic ring on a piezoelectric substrate is controlled with electrically induced anisotropic strains. Two perpendicular ferroelectric strains imposed on the ring produce magnetoelastic energy sufficient to overcome the magnetic energy barrier and reorient the magnetic state along the new easy axis of magnetization. A $45^{\circ}$ rotation of the magnetic onion state is achieved and is measured by Photo-Emission Electron Microscopy (PEEM), marking the first demonstration of deterministic control of magnetization using multiferroic rings.
\end{abstract}

\section{INTRODUCTION}

Recent resurgence of interest in multiferroics has focused on the potential of maximizing the magnetoelectric effect [1-4], where electrostatic fields can be used to control magnetization at the nanoscale. One method to achieve strong multiferroic properties is to develop laminate structures of pieozoelectric and magnetostrictive materials, coupling via strain (Figure 1). Electrically induced strain in an electrostrictive material is transferred to a magnetostrictive material, which changes magnetoelastic energy. As such, the laminate structure (Figure 2 (a)) enables magnetic control of devices $\sim 1 \mu \mathrm{m}$ or smaller, a size range where typical micro electromagnets face issues with power dissipation due to scaling of coil resistance.

More recently, ferromagnetic ring elements have been studied for their symmetric shape (yielding well-defined and stable magnetic states), as well as their varied magnetic states [5]. One interesting magnetic state available to the ring geometry is the magnetic "onion" state, which is observed at the micron- or submicron-scale (Figure 2 (b)). A remnant onion state can be formed under certain geometric and material constraints by applying a saturating magnetic field along an in-plane direction and then releasing the applied field [6]. The onion state possesses two opposite circular magnetizations, and their direction depends on the previous magnetization process. Due to its ability to maintain a magnetic state with no applied field, a few studies have attempted to manipulate the onion state for possible application in magnetic memory [7]. Reorientation of the onion state requires energy to overcome an energy barrier at its energy minimum [8].

Previously, feasibility of manipulating magnetic onion states was demonstrated in $2 \mu \mathrm{m}$ ferromagnetic $\mathrm{Ni}$ rings on a piezoelectric PMN-PT substrate. The rings were initialized by a magnetic field applied in the [011] direction on the PMN-PT substrate, and $90^{\circ}$ rotation of the onion state measured by Magnetic Force Microscopy (MFM) when electric field was applied [8].

In this study, we deposit the $2 \mu \mathrm{m} \mathrm{Ni}$ ring structures on an unpoled PMN-PT substrate, pole the PMN-PT, and then initialize the onion state at $45^{\circ}$ to the [011] direction and demonstrate $45^{\circ}$ rotation of the magnetic onion state to the [100] direction. Unlike

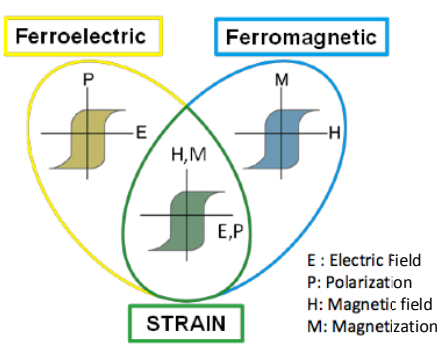

Figure 1. Concept of strain-coupled magnetoelectric structure [9].

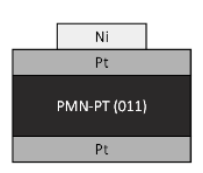

(a)

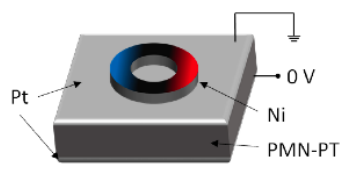

(c)

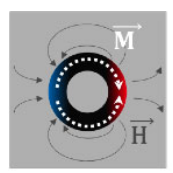

(b)

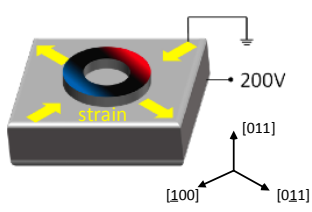

Figure 2. (a) Schematic of a Ni ring/PMN-PT heterostructure (b) Representaion of the maganetic onion state. (The white and gray arrows indicate the magnetization and the stay magnetic field, respectively.) (c) Rotation of the onion state. Left: initial onion state before applying E-field. Right: Rotated onion state after applying the E-field

the un-poled PMN-PT of the prior work, the strain on the poled PMN-PT is deterministically controllable, and the direction of the rotation is predictable. The measurement of the magnetic states is performed with PEEM.

Both MFM and PEEM are experimental methods to measure magnetization (Figure 3). MFM utilizes a magnetized scanning-probe and measures the magnetic interaction (repulsion and attraction) between the probe and the sample. Therefore, MFM is an indirect measurement of straying magnetic field from the sample. The magnetized tip may disturb magnetization in the sample. On the contrary, PEEM utilizes right and left circularly polarized x-rays, emitting secondary electrons from the sample. The emitted secondary electrons are collected and analyzed by electron microscopy to determine their spin and orbital magnetic moment. PEEM provides a direct measurement of magnetization and as a non-invasive technique has no potential to generate magnetic disturbance. PEEM can resolve magnetization to $\sim 30 \mathrm{~nm}$ of magnetization, which makes it suitable for characterization of sub-micron magnetic structures. 


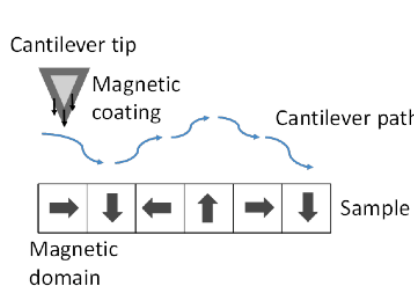

(a)

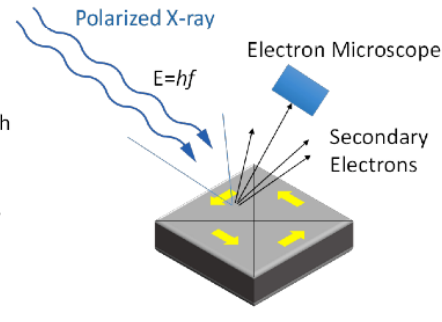

(b)
Figure 3. (a) Magnetic force microscopy and (b) photoemission electron microscopy

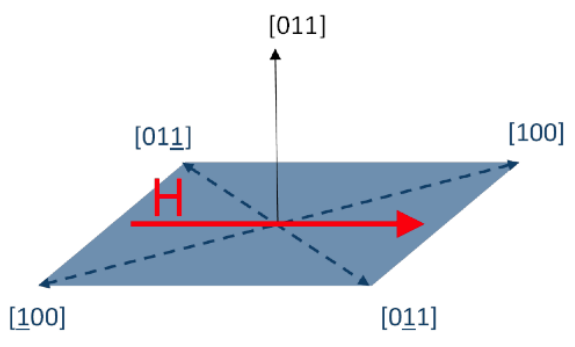

(a)

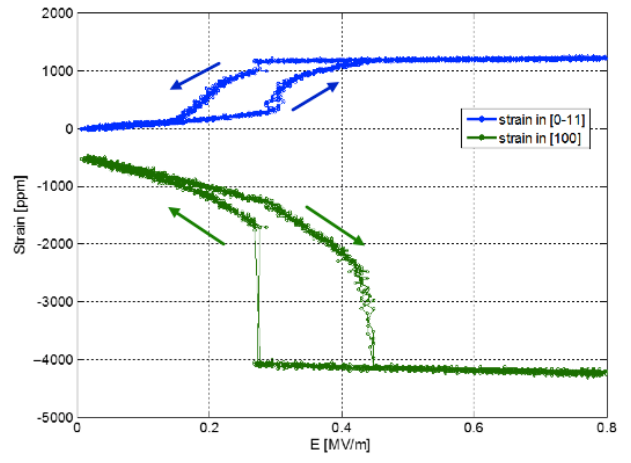

(b)

Figure 4. (a) Orientation of PMN-PT (The red arrow indicates the initialization direction of the magnetic field (H-field)) (b) PMN-PT strain curve with electric field

\section{THEORY}

\section{PMN-PT}

A single crystal piezoelectric material, (011) $\left.\mathrm{Pb}\left(\mathrm{Mg}_{1 / 3} \mathrm{Nb}_{2 / 3}\right) \mathrm{O}_{3}\right]_{(1-\mathrm{x})}-\left[\mathrm{PbTiO}_{3}\right]_{\mathrm{x}} \mathrm{x} \approx 0.34$ (PMN-PT), is chosen to generate anisotropic strains. The (011) PMN-PT produces tensile and compressive strains in two perpendicular in-plane directions [10], [011] and [100], respectively, in response to application of an out-of-plane electric field in the polarization direction ([011] direction). An out-of-plane strain is also generated with application of this electric field, but it is not transferred to the ring. The orientation and strain curve of a PMN-PT substrate are shown in Figure 4. The strain jump in Figure 4 (b) is associated with an electric field induced phase transition.

\section{Magnetic Energy Minimization Process}

The magnetic onion state is a domain structure that minimizes magnetic energy.. The total magnetic energy density is the sum of

$$
E_{\text {tot }}=E_{\text {demag }}+E_{\text {exch }}+E_{\text {aniso }}+E_{\text {Zeeman }}+E_{\text {me }}
$$

where $\mathrm{E}_{\mathrm{demag}}, \mathrm{E}_{\text {exch }}, \mathrm{E}_{\text {aniso }}, \mathrm{E}_{\text {Zeeman }}$, and $\mathrm{E}_{\mathrm{me}}$ are demagnetization energy, exchange energy, magneto-crystalline anisotropy energy, Zeeman energy and magnetoelastic energy, respectively.

After removal of the external magnetic field, the uniformly magnetized ring carries an excess of demagnetization energy resulting from the stray field generated the magnetization that is not parallel to the ring perimeter. As a result, the demagnetization energy decreases by reorienting the magnetization and forming domains at the expense of exchange energy. Rebalancing between energy terms continues until the total energy is minimized, resulting in the onion state with two domain walls. It should be noted that there exist multiple magnetic states other than the magnetic onion state, such as the vortex state and further multi-domain states [11]. The width and thickness of the ring largely determine its magnetic state at the micron-scale and nanoscale [6, 11]. Crystal anisotropy energy and Zeeman energy are assumed to be zero because (1) the evaporated Ni structure used for experiments is polycrystalline, and (2) no external magnetic field exists after the first initialization of the magnetic onion state [12]. Demagnetization energy is determined by the interaction of magnetization elements and the geometry of the ring. Exchange energy depends on neighbor magnetizations and favors uniformly aligned magnetization. Magnetoelastic energy from an external strain is given as [12]

$$
\mathrm{E}_{\mathrm{me}}=-\frac{3}{2} \lambda_{s} \mathrm{E} \varepsilon_{\mathrm{ext}}\left(\cos ^{2} \theta-\frac{1}{3}\right)
$$

where $\lambda_{S}$ and $E$ are saturation magnetostriction and Young's modulus, respectively. $\theta$ is the angle between magnetization and strain. For polycrystalline $\mathrm{Ni}, \lambda_{S}=-34 \mathrm{ppm}$, and $\mathrm{E}=200 \mathrm{GPa}$ and this was recently confirmed to hold for thin films [13]. A PMN-PT substrate with electric field along [011] direction generates two perpendicular anisotropic strains: tensile strain in [011] and compressive strain [100]. Total magnetoelastic energy can be expressed as

$$
\mathrm{E}_{\mathrm{me}}=-\frac{3}{2} \lambda_{s} \mathrm{E}\left\{\varepsilon_{[100]}\left(\cos ^{2} \theta-\frac{1}{3}\right)+\varepsilon_{[0-11]}\left(\sin ^{2} \theta-\frac{1}{3}\right)\right\}
$$

Since nickel has a negative magnetostriction, magnetic energy has a minimum along a compressive direction. In other words, anisotropic strains induce a new easy axis along the compressive direction. Reorientation of the magnetic state occurs if magnetoelastic energy from external strains produces enough energy to break the balance between energy terms, or overcome the energy barrier at the current energy minimum.

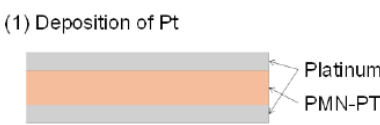

(2) Double layers of MMA

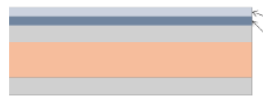

(3) E-beam Lithography

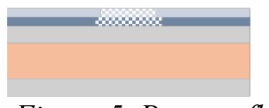

Figure 5. Process flow of Ni ring / PMN-PT magnetoelectric

heterostructure fabrication

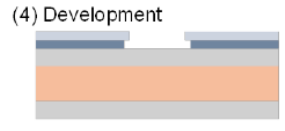

\section{PMMA 950} MMA EL-6

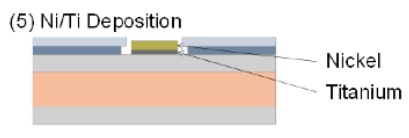

(6) Lift-off Process

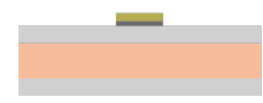




\section{EXPERIMENTAL DETAIL}

\section{Fabrication}

To investigate the strain-mediated magnetoelectric effect, we fabricated a nickel ring structure on a single crystal (011) PMN-PT substrate, where nickel is magnetoelastic and PMN-PT is piezoelectric. $5 \mathrm{~nm} \mathrm{Ti} / 50 \mathrm{~nm} \mathrm{Pt}$ is deposited on the top and bottom surface of the $8.0 \times 8.0 \times 0.5 \mathrm{~mm}^{3}$ PMN-PT as an adhesion layer/electrode. On the top (011) PMN-PT surface, double layers of methyl methacrylate (MMA) for a lift-off process are spin-coated and patterned via electron beam lithography. After the pattern development in a methyl isobutyl ketone (MIBK) solution, $5 \mathrm{~nm} \mathrm{Ti} / 30 \mathrm{~nm} \mathrm{Ni}$ as an adhesion/ferromagnetic layer is deposited by electron beam evaporation. A lift-off process is performed to define the Ni ring structures on the substrate. The dimensions of rings in this work are $2 \mu \mathrm{m} / 1.6 \mu \mathrm{m}$ and $2 \mu \mathrm{m} / 1.4 \mu \mathrm{m}$ (outer diameter/ inner diameter). The SEM images of the rings are shown in Figure 6.

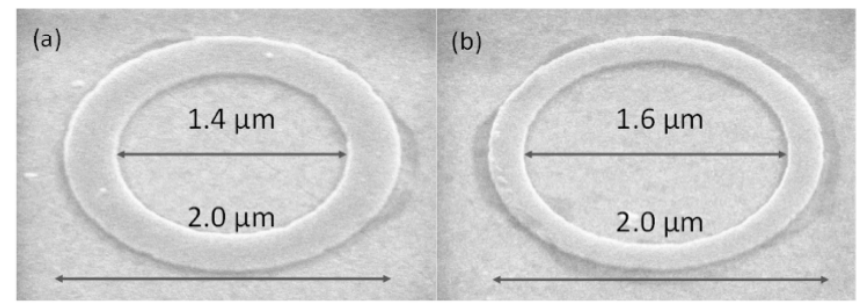

Figure 6. SEM images of nickel rings. (a) $2 u m / 1.6 \mu \mathrm{m}$ (b) $2 \mathrm{um} / 1.4$ $\mu m$. Note the images was taken under a tilt angle leading to the seemingly asymmetric shape.

\section{Measurement}

Polarization of intrinsic PMN-PT is randomly ordered. By applying an electric field across the PMN-PT, we pole, or align, the polarization to initialize in-plane strains as shown in Figure 4 (b).

After initializing the remnant strain, the onion states in the rings were formed through application of a $3 \mathrm{kOe}$ magnetic field oriented $45^{\circ}$ from the [100] and [011] directions to obtain deterministic control of magnetization. PEEM in the Lawrence Berkeley National Lab was used to measure the magnetic state of the rings. The top electrode was grounded to avoid affecting the trajectory of emitted electrons. The voltage was applied to the bottom electrode to create electric field across the PMN-PT substrate through the in-situ voltage setup in the PEEM chamber. A schematic of the device to be tested is shown in Figure 2 (c). The magnetic state in the rings was imaged as the electric field was increased.

\section{RESULTS}

After the external magnetic field of $3 \mathrm{kOe}$ was removed, the initial magnetic onion state in the rings was created (first column in Figure 7). The onion states exhibit two opposite circular magnetizations heading toward the $+x$ axis and magnetic domains emerging from the ring where at the left and right sides.

At an electric field of $0.20 \mathrm{MV} / \mathrm{m}$ (second column in Figure 7), the PMN-PT has a strain of $-1000 \mathrm{ppm}$ in [100] and $+150 \mathrm{ppm}$ in [011]. The result does not yet show appreciable magnetic change, indicating that magnetoelastic energy from anisotropic strains is not yet enough to overcome the energy barrier at its current energy minimum.

The onion state started to change at $0.40 \mathrm{MV} / \mathrm{m}$ (third column in Figure 7). The ring of $2.0 \mu \mathrm{m} / 1.4 \mu \mathrm{m}$ exhibited a more significant change than the ring of $2.0 \mu \mathrm{m} / 1.6 \mu \mathrm{m}$, which started reorienting at $0.44 \mathrm{MV} / \mathrm{m}$. The difference in magnetization between the two rings was attributed to the difference in demagnetization energy. The ring with a wider width has a smaller in-plane demagnetization factor than one with a thinner width (where ring width is defined as half the difference between inner and outer diameter). Furthermore in narrower rings the domain wall pinning is stronger due to a stronger interaction with edge roughness that plays a relatively larger role compared to the width. Thus it is easier to reorient magnetization in the wider ring, requiring less energy.

At an electric field of $0.52 \mathrm{MV} / \mathrm{m}$, rotation of the onion states was accomplished. The onion states were reoriented, or rotated, along the new easy axis, the [100] direction, which had a compressive strain. The tensile and compressive strains produced anisotropic magnetoelastic energy gradient minimizing magnetic energy in the compressive direction and magnetization was reoriented in that direction. Evidence of anisotropic strain energy creates a new easy axis of magnetization, allowing for rotation of the onion state. No further change was observed at higher voltages.

(a)
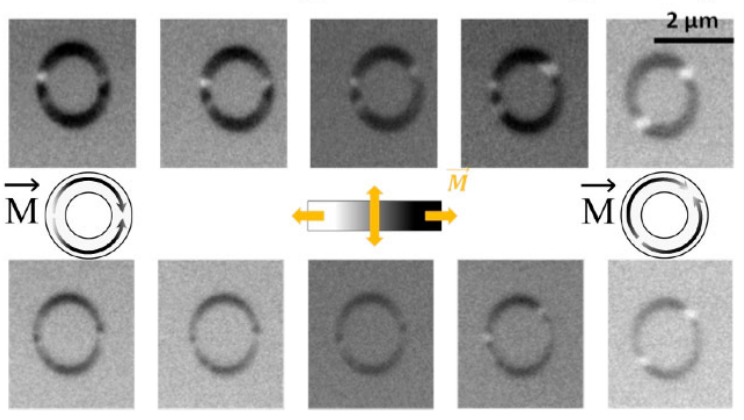

$0.0 \mathrm{MV} / \mathrm{m}$
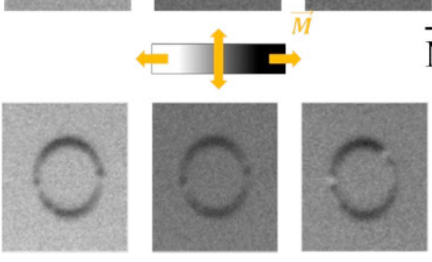

$0.20 \mathrm{MV} / \mathrm{m}$

$0.40 \mathrm{MV} / \mathrm{m}$

$0.44 \mathrm{MV} / \mathrm{m} \quad 0.52 \mathrm{MV} / \mathrm{m}$

Figure 7. PEEM images of nickel rings with different E-fields. (a) 2 $\mu \mathrm{m} / 1.6 \mu \mathrm{m}$ (b) $2 \mu \mathrm{m} / 1.4 \mu \mathrm{m}$

\section{CONCLUSION}

We demonstrated that electrically induced anisotropic strain from a piezoelectric substrate can be used to reliably rotate the magnetic onion states in magnetoleastic rings. Since only electrostatically induced strains were required to reorient magnetic states, applications requiring low power and non-volatile control of magnetization, such as magnetic memory, become possible. With further development of full $360^{\circ}$ rotation, magnetoelectric heterostructures could be used to build miniature motor systems with extremely high power densities.

\section{ACKNOWLEDGMENTS}

This project was supported by the National Science Foundation through the Cooperative Agreement Award EEC-1160504. The award, managed by Dr. Deborah J. Jackson, is for the Engineering Research Center on Translational Applications of Nanoscale Multiferroic Systems (TANMS). Fabrication was performed in Integrated Systems Nanofabrication Cleanroom (ISNC) and Nanoelectronics Research Facility (NRF) at UCLA.

\section{REFERENCES}

[1] S.-W. Cheong and M. Mostovoy, "Multiferroics: a magnetic twist for ferroelectricity," Nature materials, vol. 6, pp. 13-20, 2007. 
[2] W. Eerenstein, N. D. Mathur, and J. F. Scott, "Multiferroic and magnetoelectric materials," Nature, vol. 442, pp. 759-65, Aug 172006.

[3] R. Ramesh and N. A. Spaldin, "Multiferroics: progress and prospects in thin films," Nature materials, vol. 6, pp. 21-29, 2007.

[4] C.-W. Nan, M. I. Bichurin, S. Dong, D. Viehland, and G. Srinivasan, "Multiferroic magnetoelectric composites: Historical perspective, status, and future directions," Journal of Applied Physics, vol. 103, p. 031101, 2008.

[5] S. Li, D. Peyrade, M. Natali, A. Lebib, Y. Chen, U. Ebels, et al., "Flux closure structures in cobalt rings," Physical Review Letters, vol. 86, p. 1102, 2001.

[6] C. A. F. Vaz, T. J. Hayward, J. Llandro, F. Schackert, D. Morecroft, J. A. C. Bland, et al., "Ferromagnetic nanorings," Journal of Physics: Condensed Matter, vol. 19, p. 255207, 2007.

[7] M. Hara, T. Kimura, and Y. Otani, "Controlled depinning of domain walls in a ferromagnetic ring circuit," Applied physics letters, vol. 90, p. 242504, 2007.

[8] J. L. Hockel, A. Bur, T. Wu, K. P. Wetzlar, and G. P. Carman, "Electric field induced magnetization rotation in patterned $\mathrm{Ni}$

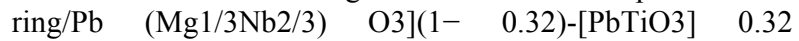
heterostructures," Applied Physics Letters, vol. 100, p. $022401,2012$.

[9] D. Khomskii, "Classifying multiferroics: Mechanisms and effects," Physics, vol. 2, pp. 1-8, 2009.

[10] T. Wu, P. Zhao, M. Bao, A. Bur, J. L. Hockel, K. Wong, et al., "Domain engineered switchable strain states in ferroelectric (011) [Pb(Mg1/3Nb2/3)O3](1-x)-[PbTiO3]x (PMN-PT, $\mathrm{x} \approx 0.32$ ) single crystals," Journal of Applied Physics, vol. 109, p. 124101, 2011.

[11] M. Kläui, "Head-to-head domain walls in magnetic nanostructures," Journal of Physics: Condensed Matter, vol. 20, p. 313001, 2008.

[12] M. Weiler, A. Brandlmaier, S. Geprägs, M. Althammer, M. Opel, C. Bihler, et al., "Voltage controlled inversion of magnetic anisotropy in a ferromagnetic thin film at room temperature," New Journal of Physics, vol. 11, p. 013021, 2009.

[13] S. Finizio, M. Foerster, M. Buzzi, B. Krüger, M. Jourdan, C. Vaz, et al., "Magnetic Anisotropy Engineering in Thin Film Ni Nanostructures by Magnetoelastic Coupling," Physical Review Applied, vol. 1, p. 021001, 2014.

\section{CONTACT}

*H. Sohn, tel: +1-310-890-7175; son0913@ucla.edu 